\title{
Establishing a head and neck unit in a developing country
}

\author{
$\mathrm{J}_{\text {ASWANI }}{ }^{1}, \mathrm{~K}$ BAIDOO $^{2}$, J OTITI $^{3}$ \\ ${ }^{1}$ Department of Surgery, School of Medicine, University of Nairobi, Kenya, ${ }^{2}$ Department of surgery, \\ University of Ghana Medical School, Accra, Ghana, and ${ }^{3}$ ENT Department, King Faisal Hospital, \\ Kigali, Rwanda
}

\begin{abstract}
Head and neck cancers pose an especially serious problem in developing countries due to late presentation requiring complex surgical intervention. These countries are faced with many challenges, ranging from insufficient health care staff to problems with peri-operative requirements, diagnostic facilities, chemoradiation services and research funding.

These challenges can be addressed through the training of head and neck surgeons and support personnel, the improvement of cancer awareness in local communities, and the establishment of dedicated head and neck institutes which focus on the special needs of head and neck cancer patients.

All these changes can best be achieved through collaborative efforts with external partners. The Karl Storz Fellowship in Advanced Head and Neck Cancer, enabling training at the University of Cape Town, South Africa, has served as a springboard towards establishing head and neck services in developing sub-Saharan African countries.
\end{abstract}

Key words: Head And Neck Cancer; Multidisciplinary; Challenges; Developing World; Africa

\section{Introduction}

Head and neck cancers are not uncommon, with approximately 640000 cases, resulting in 350000 deaths, annually worldwide. ${ }^{1}$ The need for head and neck services is therefore enormous, and especially so in developing countries. The head and neck region requires special anatomical, functional and cosmetic considerations. Therefore, a multidisciplinary approach is required, involving the disciplines of ENT, head and neck, maxillofacial, plastic, and reconstructive surgery, as well as radiation oncology, neurosurgical radiology, pathology and ophthalmology.

For a long time, head and neck surgery practice in sub-Saharan Africa has lagged behind modern standards, mainly due to lack of expertise in the field as well as inadequate support from other subspecialties such as speech therapy, reconstructive surgery and radiation oncology. ${ }^{2}$ Traditionally, because of the small number of head and neck surgeons in Africa, head and neck conditions have been managed by general surgeons without any specific head and neck training. In many situations, ENT surgeons simply diagnose head and neck malignancies and then pass patients on to the radiation oncologists for definitive management. This type of arrangement has several disadvantages, including: inadequate or inappropriate assessment and management, due to each clinical discipline working independently; lack of standardisation of management protocols, since similar conditions are managed by different specialties; and difficulties in assessing treatment outcomes.

These disadvantages can be largely overcome by establishing multidisciplinary head and neck surgical services. In this article, we review our own experience of establishing such services in Africa.

\section{Challenges of establishing head and neck surgery in a developing country}

\section{Personnel}

The ideal multidisciplinary head and neck team will include head and neck surgeons, plastic and reconstructive surgeons, maxillofacial surgeons, anaesthetists, medical and radiation oncologists, radiologists, pathologists, oncology nurses, nutritionists, speech and language pathologists, oncology social workers, physiotherapists, and occupational therapists. Most developing countries do not have sufficient numbers of these personnel. ${ }^{2}$ In Ghana, for example, there are very few specialists, thereby forcing the country to 
call on retirees to step in. The participation of plastic surgeons in the surgical treatment of head and neck cancer markedly reduces the time needed for reconstruction and also improves the cosmetic outcome. In Uganda and Rwanda, the few plastic surgeons available tend, for unknown reasons, to dedicate most of their time to other units, such as burns and craniofacial facilities. Therefore, head and neck surgeons must often resort to the use of local and pedicled flaps, even in cases in which a free flap would be ideal.

The shortage of nursing staff is also problematic. In some establishments, one surgical nurse may work for 12 hours without relief. This causes reluctance among nurses to work in the head and neck surgery theatre. Post-operative nursing care is also a challenge, with inadequate numbers of ward nurses on each shift. In Kenya, the nurse:patient ratio in the ENT ward is about 1:20 - the recommended ratio is 1:6.

Africa has very few speech and language pathologists and most are in private practice, citing as their reason lack of facilities and poor remuneration in public health institutions. ${ }^{2}$

\section{Peri-operative requirements}

Lack of safe blood for transfusion is a major limiting factor for head and neck surgery. Surgical procedures are frequently postponed due to lack of blood, especially when a free flap is planned. The shortage is largely due to dwindling numbers of willing blood donors; potential donors tend to stay away because they believe their HIV status will be disclosed to medical staff following blood donation.

Lack of basic surgical equipment, such as appropriate sutures, suction drains and sterile linen, is common.

Furthermore, scheduled operations are commonly cancelled due to breakdown of hospital and operating theatre equipment such as autoclaves, washing machines, suction machines and diathermy apparatus.

Finally, few African hospitals have sufficient intensive care unit beds and facilities to serve their country's needs. In Kenya, for example, the 1800 bed national referral and teaching hospital has a 21 bed intensive care unit. This is far below the requirements of the surgical disciplines alone.

\section{Patient factors}

Developing countries have a unique spectrum of cancer patients who usually present with advanced disease. Poverty, ignorance, poor infrastructure, long surgical waiting times, and yet to be determined patient and clinician factors may be contributory. ${ }^{3}$ Late presentation carries with it the challenge of more difficult reconstruction. Comorbid diseases such as diabetes mellitus, hypertension, human immunodeficiency virus infection (HIV) and hepatitis $\mathrm{C}$ are prevalent in many parts of sub-Saharan Africa and are often poorly managed. These comorbidities, compounded by their concomitant anaesthetic risks and the unavailability of intensive care unit beds, make intraand post-operative care challenging.

\section{Diagnostic facilities}

Computed tomography, magnetic resonance imaging and interventional radiological procedures are important elements of head and neck cancer treatment. These facilities are scanty in most developing countries, resulting in prohibitive costs and long waiting periods. Pathology services in developing countries are inadequate, with a biopsy report taking an average of three weeks in public hospitals in Kenya. Frozen section analysis, an important part of head and neck cancer surgery, is not always available. This means that it may be impossible to determine the status of surgical margins before starting reconstruction.

\section{Nutritional support}

Lack of good nutritional support for patients who are already malnourished from the disease process does not augur well for head and neck patients. In some cases, nutrients are not available in a suitable form for patients with potential swallowing difficulties. Percutaneous endoscopic gastrostomy services would be a boost in this area, if only they were affordable, readily available, and acceptable to head and neck patients.

\section{Chemoradiation services}

With the exception of a few countries, obsolete radiotherapy machines are the mainstay of radiotherapy in Africa. ${ }^{2}$ Breakdowns and malfunction are frequent, leading to delays and interruptions in post-operative radiotherapy. The use of rudimentary methods for patient planning results in geographical omissions and poor treatment outcomes. Chemotherapy is unaffordable for the average African cancer patient and the drugs are frequently unavailable, leading to their exclusion from treatment regimes.

\section{Research funding}

Research is a critical part of head and neck cancer care, and ranges from simple analysis of institutional treatment outcomes and epidemiology through to randomised, controlled studies. However, there is a lack of interest in, and poor funding of, clinical research in many institutions; hence, surgeons resort to funding their research from their own meagre resources.

\section{Strategies for overcoming these challenges}

\section{Establishment of head and neck institutes}

Dedicated head and neck institutes which were allowed to function as semi-independent units would not have to share operating theatre space and other facilities with general patients. This would increase the number of operations performed, reduce patient waiting times and improve staff skills. It may also be possible for institutes to lobby for funding as 
specialised units and to channel supplies specifically to head and neck cancer care. This would require lobbying health care policy makers and management, following careful analysis of the cost of optimal treatment in a specialist unit.

\section{International collaboration}

Through collaboration with the international head and neck surgical community, it may be possible to equip head and neck units in Africa as well as to mentor local surgeons in order to attain excellence in head and neck cancer care and research. This may also open up opportunities for short attachments and longer training placements overseas, in order to develop specific skills. Collaborating overseas institutions would also benefit from staff exchanges by exposing their staff to the different pathologies found in Africa. Opportunities for collaborative research would also be mutually beneficial.

\section{Cancer awareness and best practice advocacy}

The head and neck surgical community and other health professionals need to be involved in cancer awareness campaigns and in developing best practice guidelines. This may assist early recognition of cancer and earlier referrals. It may also provide a boost for cancer care support lobbying.

\section{Support personnel training}

As part of the development of African head and neck cancer services, there is a need to identify training programmes within Africa where support personnel such as speech and language therapists can be trained, as most countries may not be able to start their own training programmes at this time.

\section{Head and neck surgeon training}

Establishing head and neck surgical services requires leadership from head and neck surgeons, of which there are very few in Africa. Regional programmes in head and neck surgery need to be started as an expansion of the Karl Storz Fellowship in Advanced Head and Neck Surgery. It would be appropriate for these programmes to be initiated by past Karl Storz fellows.

\section{The Karl Storz Fellowship in Advanced Head and Neck Surgery}

Various initiatives have previously been made in an attempt to bridge the medical training gap between the Western world and sub-Saharan Africa, involving courses, conferences and visiting faculty. However, there had previously been no formal training in head and neck surgery, until the introduction of the Karl Storz Fellowship in Advanced Head and Neck Surgery, courtesy of Mrs Storz. This is a one-year clinical fellowship offered since 2005 by the University of Cape Town, South Africa. It is sponsored by Karl Storz Endoscopy and is open to ENT surgeons from African countries other than South Africa. It is centred principally in the otorhinolaryngology division of the University of Cape Town but also includes training in thyroid and parathyroid surgery and in maxillofacial surgery, within the departments of general surgery and maxillofacial surgery, respectively.

The Fellowship is a one-year, 'hands-on' apprenticeship, with the fellow being employed as a full-time supernumerary in the otorhinolaryngology division. The division has a strong academic and research tradition, comparable to what one sees in developed countries. South Africa, and particularly Cape Town, ranks higher than an average developing country in terms of available health facilities and funding. This provides an opportunity for fellows to practice in a developing country environment which nevertheless offers world class treatment, thus encouraging them to reproduce the same in their respective countries. The disease and patient profile in Cape Town is diverse, thus preparing the fellow to work in any country, irrespective of its status.

The Fellowship does have some limitations. There is limited exposure to skull base surgery and to the practical techniques of reconstructive surgery, and this may be a particular drawback for surgeons from countries where there are no specialists trained in these areas. Furthermore, it is sometimes challenging for the fellow to strike a balance between rotations in head and neck surgery, other otolaryngology subspecialties, thyroid and parathyroid surgery, and maxillofacial surgery. Finally, although fellows have support to relocate their entire family during fellowship training, this is not always practical since it may interrupt their children's schooling as well as their spouse's employment.

\section{- Head and neck cancer is a major public} health issue in developing countries

- Disease usually presents at an advanced stage

- The establishment of head and neck units faces considerable challenges

- Training of key team members is crucial

- The new Karl Storz Fellowship in Advanced Head and Neck Cancer (University of Cape Town) aims to address these issues

The authors of this article were among the first of seven beneficiaries of this programme. Following completion of their training, they have all returned to their own academic training centres and have attempted to replicate the clinical conditions they experienced in South Africa. The above-mentioned challenges notwithstanding, all have joined together with other specialists in their respective countries to establish multidisciplinary clinics for head and neck oncology. Among the objectives of these clinics are: (1) to improve outcomes and quality of life for patients with head and neck cancer through provision of high quality diagnostic and therapeutic services; (2) to increase interdisciplinary 
cooperation in the management of head and neck cancer, through strengthening the membership and participation of interdisciplinary teams within the multidisciplinary clinic; (3) to provide prompt intervention for patients with head and neck cancer; (4) to train and to transfer skills to specialists within the interdisciplinary teams, in order to achieve improved patient care and subsequent patient management in peripheral units; and (5) to review equipment needs for head and neck cancer management, for the involved disciplines.

The introduction of the Karl Storz Fellowship has been a great step in advancing head and neck cancer surgery in Africa. More funding could assist beneficiaries of this training to equip their home units upon completion of their fellowship, to ensure that they retain and develop their surgical skills, and to put them in a good position to establish centres of excellence where they can teach others what they have learned.

\section{Conclusion}

The need for head and neck services in developing countries is enormous. Although such countries are at varying stages of development in health service delivery, the key challenges to the establishment of functional head and neck services are shared. A multidisciplinary team approach is essential to a well functioning head and neck unit. When setting up such a service, having in place a lead team member specifically trained in head and neck surgery is a viable option, as evidenced by the Karl Storz programme at the University of Cape Town.

References

1 Parkin DM, Bray F, Ferlay J, Pisani P. Global Cancer Statistics 2002. CA Cancer J Clin 2005;55:74-108

2 Fagan JJ, Jacobs M. Survey of ENT services in Africa: need for a comprehensive intervention. Global Health Action vol 2 (2009). Available from: http://journals.sfu.ca/coaction/index. $\mathrm{phb} / \mathrm{gha} /$ article/view/1932

3 Onyango JF, Macharia JM. Delays in diagnosis, referral and management of head and neck cancer at Kenyatta National Hospital. East Afr Med J 2006;83:85-91

Address for correspondence:

Dr Joyce Aswani,

Department of Surgery,

College of Health Sciences, University of Nairobi,

PO Box 19676 - 00202, Kenyatta National Hospital,

Nairobi, Kenya

Fax: +254202710068

E-mail: j.aswani@uon.ac.ke

Dr J Aswani takes responsibility for the integrity of the content of the paper

Competing interests: None declared 